\title{
AB OSTIO FLUMINIS ANAE...
}

\author{
M. BEndAla GaláN
}

La vía 23 del Itinerario de Antonino - ab ostio fluminis Anae Emeritam-, que enlaza la desembocadura del Guadiana con Emerita Augusta pasando por Itálica, presenta aún el problema geográfico de la reducción satisfactoria de sus dos primeras mansiones: Praesidium y Ad Rubras (1). Desde la mansio siguiente, Onoba (Huelva), el trazado de la vía puede seguirse sin dificultad por Ilipla (Niebla), Tucci (Tejada, Escacena), hasta Itálica, donde tuerce hacia el norte para dirigirse a Mérida.

Las numerosas propuestas de ubicación de las dos problemáticas mansiones pueden agruparse en dos tendencias: la que las sitúa muy al interior de la provincia de Huelva, determinando, por tanto, un trazado serrano para el tramo viario que les corresponde, y la que se decanta por un recorrido próximo a la costa, más o menos coincidente con la carretera moderna de Ayamonte a Huelva. En este último caso, Praesidio y Ad Rubras debían estar donde los pueblos actuales de Lepe y Cartaya, y la vía debía pasar el río Odiel a la altura de Gibraleón para dirigirse a Huelva.

La hipótesis del trazado costero, mantenida hace tiempo por autores como Saavedra y A. Blázquez, goza en la actualidad de bastante aceptación. La ha defendido últimamente J. M. Luzón (2), buen conocedor de la provincia de Huelva, y es el trazado sugerido por J. M. Roldán (3) en su estudio de conjunto de las vías

(1) Surgió mi interés por esta vía onubense durante el tiempo en que formaba parte, como profesor, del Departamento de Prehistoria y Arqueología de la Universidad de Sevilla, por los años 1975 y 1976 . Se me encomendó entonces redactar la parte correspondiente a la Arqueología para el Catálogo Monumental de Huelva, que patrocinaba la Excma. Diputación de esa provincia. Redactado y entregado el primer volumen, está aún por editar. Pero como parte del trabajo de campo entonces acometido, recorrí buena parte de la provincia con Alfonso Jiménez Martín, miembro del equipo encargado del Catálogo, hoy Catedrático de Análisis de Formas de la E.T.S. de Arquitectura de Sevilla. A él, buen conocedor de la provincia de Huelva, y arqueólogo de vocación, le debo sugerencias sustanciales a la hora de emprender este estudio. De esta forma, lo que en buena medida empezó en un marco de amistad y de colaboración científica, termina en otro acto de amistad, en una modesta contribución al homenaje que justamente se rinde a la memoria de D. Gratiniano Nieto. Séalo también de postrera colaboración científica al cabo de una larga convivencia en el Departamento de Prehistoria y Arqueología de la Universidad Autónoma de Madrid.

(2) Luzón Nogué, J. M.: "Antigüedades romanas en la provincia de Huelva», en Huelva: Prehistoria y Antigüedad, Madrid, 1974, pág. 274.

(3) Roldán Hervás, J. M.: Itineraria Hispana. Fuentes antiguas para el estudio de las vías romanas en la Península Ibérica, Valladolid-Granada, 1975, pág. 81, lám. III. 
hispanas (4). Pero la posibilidad de un trazado costero encuentra tales dificultades que obliga, a nuestro entender, a prescindir de ella. En primer lugar, no se corresponde con las distancias consignadas en el Itinerario de Antonino, cuyo grado de fiabilidad está bien probado, y sólo cuando se tienen pruebas suficientes en contrario deben desestimarse sus datos (5). En efecto, si se supone que Praesidio es Lepe y Ad Rubras Cartaya, las distancias establecidas en el Itinerario -24 millas $(35,5 \mathrm{Km}$.) desde la desembocadura del Guadiana a Praesidio y 28 millas $(41,4 \mathrm{Km}$.) desde Praesidio a Ad Rubras- son muy superiores a las de Ayamonte a Lepe $(22 \mathrm{Km}$.) y de Lepe a Cartaya $(5,5 \mathrm{Km}$.). La diferencia global, más de $49 \mathrm{Km}$. (de las 52 millas $/ 76,9 \mathrm{Km}$. del Itinerario a los $27,5 \mathrm{Km}$. de la carretera moderna), sólo tendría explicación si se admite que el Itinerario está equivocado en las dos distancias, a no ser que se trate de buscar equivalencias particulares a la milla en este tramo, como en otros casos se ha pretendido infundadamente.

No conviene olvidar, por otra parte, que una vía cercana a la costa tendría que atravesar terrenos poco practicables, con zonas de esteros y marismas como las del entorno de Isla Cristina y la cuenca del río Piedras, obstáculos que debían de ser aún más difícilmente franqueables en la Antigüedad. No existen, además, pruebas arqueológicas que permitan apoyar la hipótesis de que Praesidio y Ad Rubras estuvieran en Lepe y Cartaya. Ni en estas localidades ni en sus cercanías han aparecido vestigios romanos de importancia, y todo lleva a pensar que este sector de la costa onubense quedó como zona muy marginal respecto de los centros de interés de la época. Los muy escasos restos romanos hallados en la zona prueban, en todo caso, que no estaba totalmente despoblada y que debieron existir pequeñas comunidades dedicadas fundamentalmente a la pesca y a las labores agrícolas (6).

La inviabilidad del camino costero invita a reconsiderar la posibilidad de que la vía se dirigiera desde la desembocadura del Guadiana hacia el interior, haciendo un recorrido más largo y ajustado a las distancias consignadas en el Itinerario. Rodrigo Caro propuso ya la identificación de Ad Rubras con Cabezas Rubias, locali-

(4) Para una revisión de las diferentes hipótesis propuestas para el tramo inicial de la vía, con las citas de los autores antiguos, ver: Hernández, F.: "El cruce del Odiel por la vía romana de Ayamonte a Mérida", $A E s p A$ 31, 1958, págs. 126 y ss.

(5) Roldán Hervás, J. M.: op. cit., pág. 33.

(6) Un pequeño asentamiento prerromano ha sido excavado en La Tiñosa (Lepe), junto a la desembocadura del Piedras. Lo ocuparon gentes dedicadas fundamentalmente a la pesca, aproximadamente durante los dos siglos inmediatamente anteriores a la conquista romana. Cf. Belén, M. y Fernández-Miranda, M.: "La Tiñosa (Lepe, Huelva)", Huelva Arqueológica 4, 1978, págs. 197 y ss. Tenemos datos de algunos hallazgos romanos en Cartaya y su entorno inmediato. Durante el derribo de una casa del pueblo se halló un fragmento de una lastra de mármol blanco, de $18 \mathrm{~cm}$. de ancho, 17 de alto y 7,5 a 10,5 de espesor (la parte posterior estaba sin regularizar, como para ser adosada), que conservaba en el frente parte de una decoración floral muy sencilla y, en el borde superior, entre líneas, la inscripción... RESYDEDO... (con nexo D-E). Fue entregada por mí al Museo Provincial de Huelva. En el lugar conocido como del "Pilar de Mogaya", a $2 \mathrm{Km}$. de Cartaya en dirección a Huelva por la carretera actual, se halló a fines de los años cincuenta un sarcófago romano de mármol, sin decoración, del que se guarda parte en un local de la iglesia parroquial del pueblo. Prospectado el lugar, no encontramos ningún otro vestigio antiguo. Hemos visto también restos de cerámicas y de tégulas romanas en una finca propiedad de D. David Pastor, situada a unos $2 \mathrm{Km}$. de la misma población, por la carretera que conduce a El Rompido. Quede constancia de mi agradecimiento al citado Sr. Pastor y a D. Guillermo Pérez por su amabilidad y sus valiosas indicaciones. 
dad onubense situada muy al norte, en el Andévalo, y el paso de la calzada por Puebla de Guzmán (7). La ecuación Ad Rubras/Cabezas Rubias ha sido, después, mantenida por numerosos autores: Ceán Bermúdez, Schulten, Thouvenot (8).

La mansion anterior, Praesidio, ha sido identificada, por aquellos autores que admiten el recorrido de la calzada tierra adentro, con Puebla de Guzmán (Ceán) o con Villanueva de los Castillejos (Miller, Thouvenot) (9). Ultimamente, A. Tovar llamó la atención sobre la relación que tal vez guardara, con la mansio del $I A$ 431,10, una pequeña fortificación - un praesidium - descubierta más al norte, ya en territorio portugués, en Mourao (10).

Pero tampoco faltan dificultades para la admisión de estas reducciones, sobre todo, de nuevo, por la falta de correspondencia con las distancias del Itinerario, esta vez por exceso. El castillo de Mourao queda demasiado al norte, y la identificación Ad Rubras/Cabezas Rubias no deja de presentar problemas, pese a la sugestiva homofonía de los topónimos. Si llevamos la calzada hasta Cabezas Rubias, la distancia desde Ayamonte (unos $77 \mathrm{Km}$.) viene a coincidir con las 52 millas del $I A$ entre la desembocadura del Guadiana y Ad Rubras, circunstancia que ha favorecido esta identificación tradicional; pero, en ese caso, las 28 millas hasta Onoba (Huelva) se quedan demasiado cortas para los casi $70 \mathrm{Km}$. que la separan de Cabezas Rubias (11). Conviene añadir, además, que los topónimos alusivos al color "rubio", "rojo" o "colorado" son frecuentes en toda la comarca serrana de Huelva por la abundancia de tierras con alto contenido en óxido de hierro.

Por todas estas razones, y partiendo de la hipótesis de que la calzada romana descrita en el Itinerario debía adentrarse hacia el interior de la provincia onubense, emprendimos prospecciones con el propósito de dar con pruebas arqueológicas de la vía y de las mansiones en discusión. Como resultado de ellas tenemos la posibilidad de proponer un nuevo recorrido para la calzada (fig. 1) y otras hipótesis que sumar a las existentes acerca de la reducción geográfica de las mansiones, lo que a continuación se expone a título provisional y por si algo aporta a la discusión científica del problema (12).

(7) Vid. Hernández, F.: op. cit., pág. 127.

(8) Tovar, A.: Iberische Landeskunde I (Baetica), Baden Baden, 1974, pág. 172. Según Madoz (Diccionario geográfico-estadístico-histórico de España...), V, 1846, 32, se descubrieron en este lugar las ruinas de un templo a Endovélico. Luzón, que discrepa de la identificación con Ad Rubras, dice haber reconocido restos de una calzada romana en las cercanías, tras el "Cabezo de la Divisa" (cf. op. cit., pág. 307). K. Miller, en su obra clásica Itineraria romana (Stuttgart, 1916; ed. anast. Roma, 1964), sitúa Ad Rubras en el Cerro del Andévalo (184 y fig. 43).

(9) Miller, K.: op. cit., 184; Thouvenot, R.: Essai sur la province romaine de Bétique, París, 1940 (reimp. 1973), pág. 488. Cerca, en la finca "La Nava", han aparecido algunos epígrafes romanos (Luzón, J. M.: op. cit., pág. 302).

(10) do Paço, A. y Baçao Leal, J.: "Castelo da Lousa, Mourao (Portugal)". Una fortificación romana de la margen izquierda del Guadiana, $A E s p A 39,1966$, págs. 167 y ss.

(11) Esta es la razón por la que Rodrigo Caro y otros autores posteriores propusieron ubicar Onoba en Gibraleón, algo más cerca de Cabezas Rubias, cosa totalmente descartada hoy, pese a que algunos restos romanos hayan aparecido en Gibraleón. Cf. Luzón, J. M.: op. cit., pág. 311; Hernández, F.: op. cit. passim.

(12) Un avance de estas investigaciones se ha publicado en Blanco, A. y Rothenberg, B.: Exploración arqueometalúrgica de Huelva, Barcelona, 1981, pág. 28, nota 13. Los resultados de las prospecciones efectuadas 


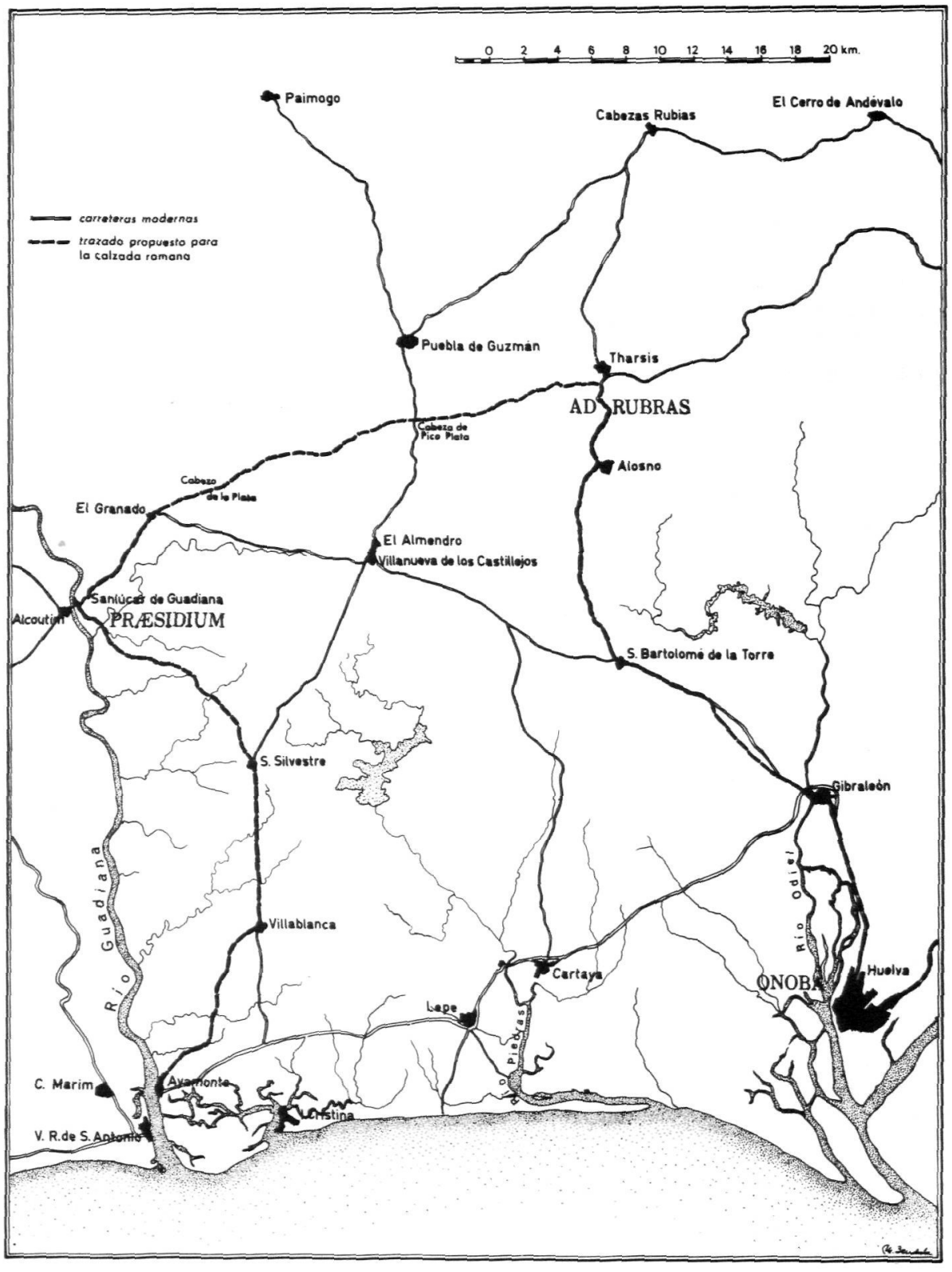




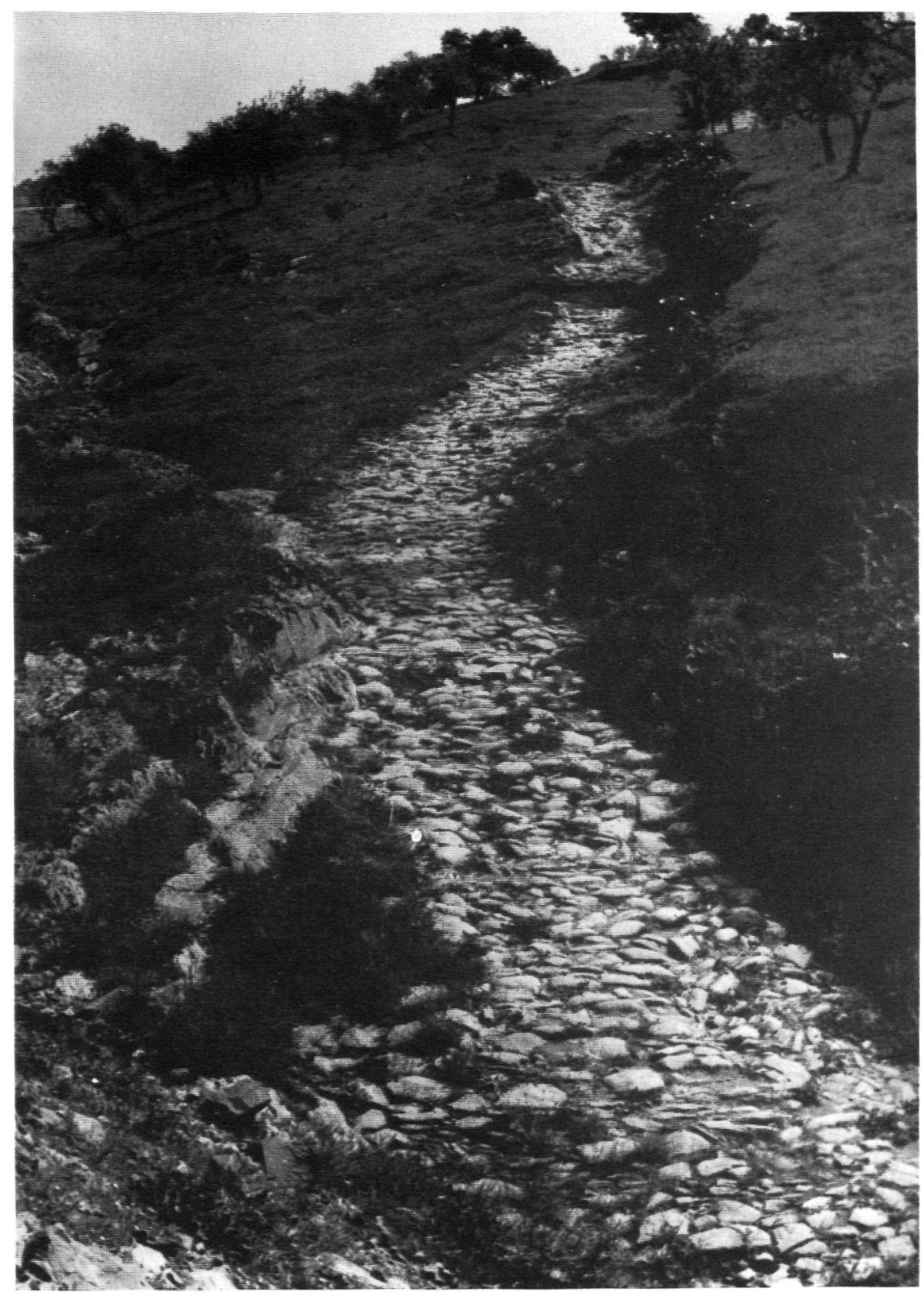

Foto 1. 


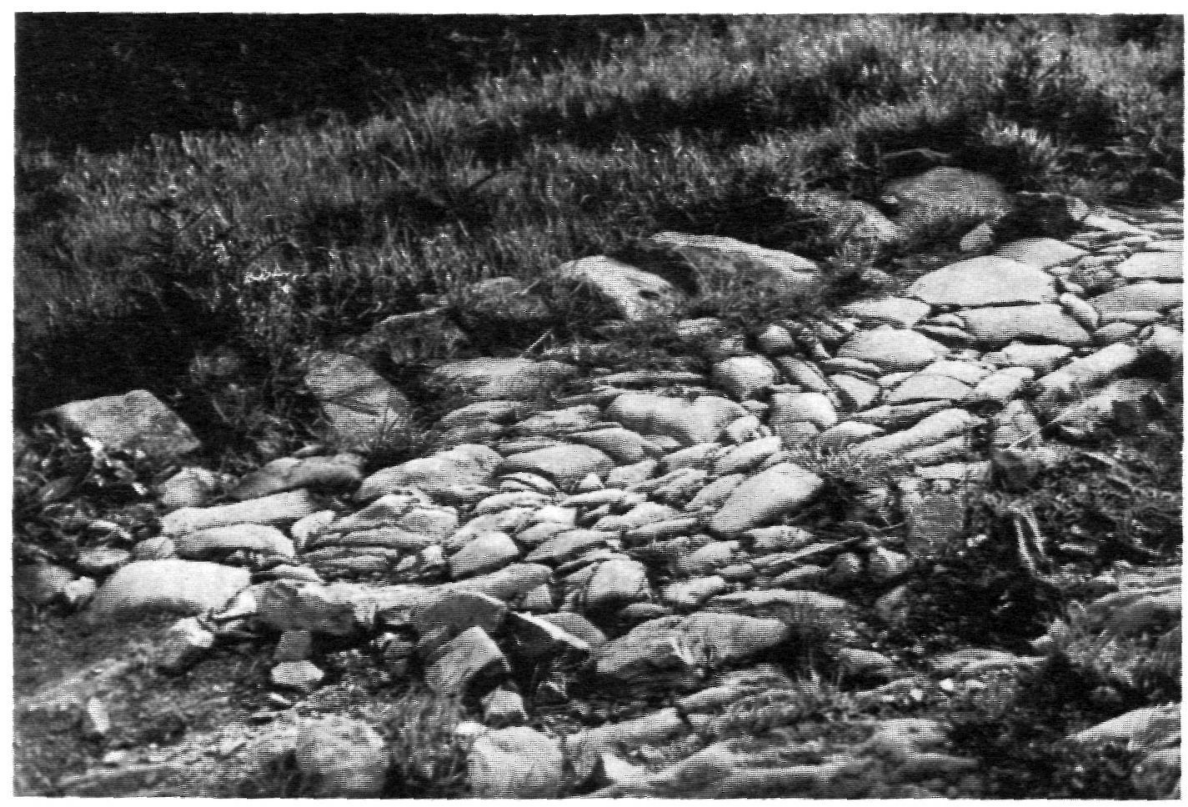

Foto 2.

Siguiendo la pista de los caminos antiguos indicados en los planos del Servicio Geográfico del Ejército (1:25.000, 1:50.000 y 1:200.000), encontramos una vieja calzada empedrada que parte de Sanlúcar de Guadiana en dirección a San Silvestre (13), conservada en un tramo de varios hectómetros en buen estado, que bien pudiera ser obra romana (foto 1). En la actualidad se le ha superpuesto parcialmente una pista moderna, que sigue en líneas generales su recorrido. La calzada antigua tiene anchura variable, en torno a 2,80 m., y se adapta a la accidentada topografía del terreno, aunque se procedió también a encajarla mediante socavado de puntos altos para evitar cuestas demasiado pronunciadas. El empedrado, bien conservado en bastantes tramos, es de caliza grisácea en su mayor parte, con piezas irregulares de diversos tamaños; son de mayores dimensiones las que delimitan los bordes (foto 2) $y$, de trecho en trecho, se dispuso una hilera transversal de piedras hincadas para sujetar mejor la pavimentación y evitar deslizamientos. Estos detalles ponen de manifiesto que se trata de una obra bastante cuidada, aunque en la actualidad ofrece un firme muy irregular por la pronunciada erosión del enlosado y del dislocamiento de muchas piezas. No se advierten huellas del paso de carruajes y debe suponerse, dadas las características generales del trazado, que se trata de una calzada destinada exclusivamente al uso a pie y con caballerías.

Podríamos estar ante los restos de una vía romana, excepcionalmente conservada en un paraje bastante apartado y poco transitado después, y lo suficientemente importante, por la envergadura de la construcción, como para hacer pensar que 
corresponda, dada su situación, a la calzada recogida en el Itinerario de Antonino. En este caso, habría que plantearse la posibilidad de que Praesidium hubiera estado donde Sanlúcar de Guadiana, situado a una distancia respecto de la desembocadura del río que se ajusta bien a las 24 millas fijadas en el Itinerario. Las noticias históricas más antiguas sobre esta Sanlúcar se remontan a comienzos del siglo XV (se la menciona como Sanlúcar de Guadiana o de Alcautín); por entonces debió de ser fundada por los señores de Gibraleón, en terrenos de su dominio, como enclave comercial en la frontera con Portugal (14). No se conocen restos en el lugar que permitan suponer que el pueblo medieval se asentara sobre un despoblado anterior. Es de destacar que el caserío, arrimado al río, queda al pie de un alto cerro sobre el que se encarama un castillo moderno (es el "Cerro del Castillo" o «Piedra del Niño de Dios"). Es un magnífico observatorio en medio del ambiente serrano en que se encuentra, y desde él se controla muy especialmente el río, con Sanlúcar en la rivera izquierda y frente, en la margen derecha, el pueblo portugués de $\mathrm{Al}$ coutim, así como la calzada descrita. Reúne, pues, todas las condiciones para que en él estuviera el castillo o praesidium destinado a la vigilancia de la vía y del entorno. Quizá hubo una pequeña fortificación romana similar a la citada de Mourao y, según curiosos del lugar, el castillo moderno, de la época de la "francesada", se construyó sobre otro anterior atribuido a los portugueses (15). Si existió el castillo romano es algo que no hemos podido comprobar con una inspección ocular del sitio, ocupado como está por la fortaleza moderna, de grandes dimensiones y ya en ruinas.

Al pie del cerro, en su parte meridional y junto al pueblo, hay un manantial con obras para dotarlo de estanques cubiertos y abrevaderos. Es de interés comprobar que la obra moderna se asienta sobre los restos de un frogón de hormigón seguramente romano. También se hallaron restos de ánforas romanas en la finca Huerta Torres, dos kilómetros al norte de Sanlúcar, junto al Guadiana. Y una tumba de inhumación de gran tamaño, junto a otros restos antiguos, aparecieron a bastantes metros de profundidad al hacer el muelle junto al río (16).

$\mathrm{Si}$ admitimos que la calzada enlosada es la del Itinerario y que Praesidio estuvo en el Cerro del Castillo de Sanlúcar, lo que parece verosímil, el primer tramo de la vía 23 del $I A$ puede reconstruirse fácilmente siguiendo, de norte a sur, el camino

en los años indicados antes, y en algunas visitas posteriores, los teníamos por provisionales, a la espera de nuevas prospecciones, difíciles en muchos casos por las características de los terrenos de la sierra, y de efectuar catas arqueológicas en la vía y otros análisis. Como por diversas circunstancias me ha sido imposible después realizar esa labor, me decido ahora a publicar las conclusiones obtenidas, a sabiendas de su provisionalidad.

(13) Corresponde al llamado "Camino de San Silvestre". Ver hoja 980 del 1:50.000 (1. a ed. 1950).

(14) Ladero Quesada, M. A.: "Los señores de Gibraleón", Cuadernos de Historia, Anexos de Hispania 7, 1977, pág. 70 .

(15) El castillo sirvió de refugio a los habitantes de la zona durante la Guerra de la Independencia. En 1665 se apoderó de la población el conde de Schomberg, gobernador de la provincia de Alentejo, según recuerda Madoz 13 (1849), 741 ss.

(16) Agradezco a D. Miguel Ferrera Ojeda, vecino de Sanlúcar de Guadiana, este último dato y la oportunidad de inspeccionar los restos anfóricos de Huerta Torres, que guarda en su poder.

(17) Ver hojas 980 y 998 del mapa 1:50.000 (1. ${ }^{\text {a }}$ ed. 1946). 
enlosado, o camino viejo de San Silvestre, hasta esta localidad; desde aquí hasta Villablanca por la actual carretera local, de trazado muy recto; y por último hasta Ayamonte, en el ostium fluminis, por el camino viejo de Ayamonte a Villablanca (17). El recorrido de unos $36 \mathrm{Km}$. se corresponde bien con la distancia de 24 millas del Itinerario.

El tramo de la calzada desde Praesidio hasta Onoba y la ubicación de Ad Rubras plantean nuevos problemas, pero puede suponerse un trazado coherente, y ajustado a las distancias del Itinerario, siguiendo los lugares con vestigios romanos, los caminos antiguos, las referencias toponímicas; pasaría por El Granado, siguiendo hacia el este algo al sur de Puebla de Guzmán y por las cercanías de Tharsis, donde cabe situar Ad Rubras; desde aquí enlazaría con Huelva por Alosno, San Bartolomé de la Torre y Gibraleón. Veamos ahora el recorrido propuesto con algún detenimiento.

Desde Praesidio/Sanlúcar debía seguir la calzada en dirección nordeste hacia El Granado, por el mismo camino de la carretera local moderna. Precisamente en El Granado, según tradición referida por Luzón, se ha ubicado también Praesidio (?), y el mismo autor recoge la noticia de que hay en el lugar restos visibles de una vía que se dirige a Cabezas Rubias (18). La calzada que nos ocupa, como antes se dijo, no puede subir tan al norte. Su recorrido hasta las cercanías de Tharsis no podemos determinarlo a falta de prospecciones metódicas en la zona, labor que no resultaría nada sencilla por lo intrincado del terreno. Pero algunos topónimos permiten sospechar que un antiguo camino enlosado se dirigía desde El Granado en dirección a Tharsis. Al nordeste de El Granado, saliendo por el llamado "Camino del Toro", y ya en término de El Almendro, se llega al "Cabezo de la Plata", elevación que domina el "Collado de la Plata», topónimos que, como bien se sabe, hacen alusión a una antigua calzada empedrada. Siguiendo la misma dirección, por el «Puerto Colorado» y la «Sierra de las Tres Piedras", se llega más al este, unos tres kilómetros al sur de Puebla de Guzmán, y ya en su término, al «Gabezo Pico Plata" (19); y prolongando el camino en el mismo sentido se llega a la zona de Tharsis.

La gran importancia económica de la cuenca minera de Tharsis en época romana, justifica sobradamente que en el Itinerario se recoja la calzada que hasta ella conduce. Las minas de Tharsis, en efecto, seguían en importancia a las de Riotinto (las escorias procedentes de labores antiguas se cuentan por millones de toneladas), y abundan los restos arqueológicos - esculturas, epígrafes, monedas, cerámicas, etc. - que delatan la presencia romana (20). La mansio Ad Rubras del Itinerario debió de estar en sus cercanías, como se desprende del uso de la preposición ad (21), mientras que el apelativo rubrae resulta adecuado a las tierras «rojas» de Tharsis. En relación con la posible ubicación de esta mansio es interesante recordar un dato citado por Luzón, la existencia junto a Alosno, muy cerca de Tharsis por el

(18) Luzón, J. M.: op. cit., pág. 311.

(19) Ver mapa 1:50.000, hoja $958\left(10^{a}\right.$ ed. 1955).

(20) Luzón, J. M.: op. cit., págs. 300-301 y 318-319; Blanco, A. y Luzón, J. M.: «Mineros antiguos españoles", AEspA 39, 1966, págs. 73 y ss.

(21) Roldán, J. M.: op. cit., págs. 31-32. 
sur, de restos de una población romana, en la que se hallaron restos de cerámicas y de tégulas y un epígrafe. Sugiere él mismo que el "Camino Viejo de Alosno", que conduce a San Bartolomé de la Torre, fuera en origen una vía romana (22).

Desde este punto, el recorrido de nuestra calzada hasta Huelva puede seguirse con bastante seguridad. Primero desde Alosno a San Bartolomé de la Torre, localidad esta última donde han aparecido abundantes restos romanos, en el mismo lugar donde se halla la torre medieval que le da nombre y en las cercanías (23). Luego, de San Bartolomé a Gibraleón por el camino antiguo, seguido por la carretera comarcal moderna, que se separa de aquél, no obstante, en largos tramos. La prospección de este sector permitió comprobar que la vía antigua se apoya en el terreno pizarroso, sin pavimentación añadida alguna, aunque en algunos tramos conserva restos de un empedrado menudo muy mal conservado. Desde Gibraleón a Huelva debía seguir la calzada el mismo camino que la carretera actual.

El paso de Odiel a la altura de Gibraleón debió hacerse por la llamada «Pasada del Zuar", un vado que Madoz tuvo por "azuda" o dique para conducir las aguas a molinos de harina. Se trata de un paso muy bien construido, consistente en un muro apoyado en el fondo rocoso, perforado con portillos abovedados para permitir el paso del agua; sostiene un tablero de cuatro metros de ancho, cuidadoamente enlosado, en el que se aprecian reparaciones de menos calidad que la obra originaria. En condiciones normales queda unos treinta centímetros por encima del agua, y en caso de crecidas quedarían aguas laminares sobre el vado que no impedirían totalmente el paso. Esta importante obra de ingeniería, hoy arruinada, permitía atravesar cómodamente el río, y con reparaciones diversas ha estado en uso hasta el siglo pasado. Según F. Hernández es obra originariamente romana, entre otras cosas por su alto nivel constructivo y por ser parte de un ambicioso proyecto viario no atendible por la villa medieval (24). Luzón no es del mismo parecer; considera más lógico que los romanos hubieran construido un puente y que los sillares de caliza usados en el vado fueran aprovechados de ese posible puente. Se plantea asimismo la posibilidad de que la calzada atravesara el río por alguno de los pasos que hay unos seis kilómetros más arriba, como el del "Rocho del Mal Paso" (25). No parece, sin embargo, que la calzada romana cruzara el Odiel tan arriba y no es descartable que el vado de Gibraleón fuera romano como proponía F. Hernández. Hemos tenido ocasión de examinar sus ruinas en condiciones particularmente favorables, en tiempos de fuerte sequía, con buena parte de la obra en seco. Es sin duda un trabajo de ingeniería de gran calidad, perfectamente adecuado al sitio y a su función, como un verdadero puente de tablero bajo y horizontal, aunque menos costoso y de pretensiones más modestas que un puente estilo Mérida. Que fuera obra romana parece probable, pero un dictamen más solvente requiere un análisis más detenido que el simple examen visual que hemos podido realizar. No hay vestigios de puente alguno, ni en el lugar del vado ni en sus proximidades, y no es

(22) Luzón, J. M.: op. cit., págs. 284 y 304.

(23) Luzón, J. M.: op. cit., pág. 317.

(24) Hernández, F.: op. cit., págs. 140-146.

(25) Luzón, J. M.: op. cit., pág. 274. 
preciso que lo hubiera si se contaba con un medio de salvar el río como el paso que comentamos (26).

Como conclusión, propongo una nueva interpretación del recorrido del tramo inicial de la vía 23 del $I A$, que, en principio, se ajusta bien a las distancias que en él se indican, con la consiguiente propuesta de ubicación de las dos primeras mansiones. Praesidio pudo estar en el Cerro del Castillo de Sanlúcar de Guadiana, y la distancia de $36 \mathrm{Km}$. a Ayamonte se ajusta bien a las 24 millas que señala el Itinerario para esta primera mansio. Si Ad Rubras se hallaba en las cercanías de Tharsis, también las distancias del Itinerario resultan ajustadas, tanto desde Sanlúcar como desde Huelva (algo más de cuarenta kilómetros en los dos casos frente a las 28 millas del Itinerario en uno y otro sentido).

Es perfectamente explicable que una calzada con el recorrido propuesto quedase reflejada en el Itinerario dada su importancia. Se trata de la vía que conduce desde la ciudad portuaria de Onoba - y antes desde Itálica y su entorno- a la rica cuenca minera de Tharsis y a un centro a orillas del Guadiana desde el que pasar, atravesado el río (es de suponer que por barcaje, como se ha hecho siempre), a otra vía importante recogida también en el Itinerario, la 22. Esta última, procedente de Esuris (Castro Marim) lleva a ciudades tan destacadas como Myrtilis (Mértola) y Pax Iulia (Beja) (27). Desde Praesidio seguía nuestra vía, por fin, hasta la desembocadura del Guadiana.

La ruta propuesta desde Huelva y Gibraleón a la raya de Portugal por Sanlúcar, hacia Mértola, es una de tantas de tradición inmemorial que debieron quedar firmemente asentadas en y desde época romana. La que ahora nos ocupa se configuró, con los cambios que son de suponer, como uno de los ejes sobre los que giró la historia de la región. En la Edad Media, la vía de Gibraleón a Portugal por San Bartolomé de la Torre, Villanueva de los Castillejos, El Granado y Sanlúcar de Guadiana, fue una de las principales arterias comerciales de la provincia de Huelva (28). En torno suyo parece configurarse el señorío de Gibraleón, que tuvo en la actividad mercantil una de sus fuentes básicas de riqueza (29); el territorio de su dominio lo constituía una franja de terreno, por el que discurre la vía, desde el Odiel y la costa atlántica por la desembocadura del Piedras, hasta la frontera de Portugal en el Guadiana, territorio limitado por el señorío de Ayamonte al oeste y las tierras del condado de Niebla por el este y el norte. La posesión de una vía

(26) Hernández, F.: op. cit., págs. 139-140, se hace eco de que en la Descripción Geográfica de Al Andalus de Ahmad al-Ràzi, se cita a Gibraleón como villa situada junto al río de Alcantir, de modo que el Odiel era llamado "río de los puentes». Para F. Hernández debe explicarse el nombre por haber tomado el vado, que debía estar entonces arruinado, por ruinas de puentes.

(27) Roldán, J. M.: op. cit., págs. 78-79. Recordemos que en la zona de Alcoutim, la población frontera a Sanlúcar de Guadiana, han aparecido también restos romanos. Cf. de Alarçao, J.: Portugal romano, Lisboa, 1973, págs. 162 y 201.

(28) González Gómez, A.: Moguer en la Baja Edad Media (1248-1538), Huelva, 1977, págs. 132 y ss., gráfico n. 6 .

(29) Pardo Rodríguez, M. ${ }^{a}$ Luisa: Huelva y Gibraleón (1282-1495). Documentos para su historia, Huelva, 1980, págs. 60 y 61 . Es de señalar el hecho infrecuente de que Gibraleón contara con una feria desde la temprana fecha de 1323. 
propia para el comercio con Portugal debió ser fundamental para evitar dependencias y servidumbres respecto del señorío de Ayamonte, motivo de continuas fricciones como revela el conflicto surgido a partir de 1414 entre Lepe, del señorío de Ayamonte, y Gibraleón, por la pertenencia de las rentas de la barca del Piedras (30).

Quede lo hasta aquí expuesto como un conjunto de hipótesis, verosímiles y con argumentos y datos que las apoyan, pero necesitadas de una investigación más menuda que habrá que emprender, sea yo mismo en mejor ocasión, sea cualquiera otro que decida hacer suya la empresa.

Madrid, septiembre de 1986

(30) Ladero Quesada, M. A.: op. cit., págs. 68-70. 